Research Article

\title{
Attitude Tracking of Rigid Spacecraft with Actuator Saturation and Fault Based on a Compound Control
}

\author{
Chunhua Cheng $(\mathbb{D}$, Hang Yang, Qian Wang, Lin Li, Qiang Han, Huan Ouyang, \\ Haiyang Ma, and Xinyi Lv
}

Qingdao Branch, Naval Aeronautic University, Qingdao 266041, China

Correspondence should be addressed to Chunhua Cheng; chch715@126.com

Received 29 October 2020; Revised 29 November 2020; Accepted 1 December 2020; Published 28 December 2020

Academic Editor: Jinyan Song

Copyright (C) 2020 Chunhua Cheng et al. This is an open access article distributed under the Creative Commons Attribution License, which permits unrestricted use, distribution, and reproduction in any medium, provided the original work is properly cited.

\begin{abstract}
A compound control based on active disturbance rejection control (ADRC) scheme and slide mode control (SMC) is proposed to investigate the attitude tracking problem for a spacecraft with modeling uncertainties, external disturbances, actuator failures, and actuator saturations simultaneously. A positive term including control input is separated from the system, and then, the active disturbance rejection concept and the extended state observer (ESO) are applied to deal with the general uncertain item caused by uncertainties, external disturbances, actuator failures, and actuator saturations. The sliding mode surface is designed to transform the attitude tracking problem into attitude stabilization problem. In order to deal with the actuator saturations, a saturation degree coefficient and its corresponding adaptive law are introduced. Compared to other existing references, the proposed scheme does not need to know the structure or upper bound information of the inertial matrix uncertainties and external disturbances. Finally, the stability of the closed-loop system is analyzed by using input to state stability theory. Simulation results are given to verify the effectiveness of the proposed scheme. More importantly, the proposed technique can also be applied to the attitude stabilization of other aircraft, such as the attitude of unmanned aerial vehicle and helicopter in maritime rescue.
\end{abstract}

\section{Introduction}

With the development of space missions, the reliable control scheme is significantly important for the rigid spacecraft. As the part of spacecraft systems, attitude control plays an important role in spacecraft design and has been studied extensively under various scenarios [1-5]. The reliability of attitude control determines that the planned missions can be accomplished successfully or not. It is a significant challenge to obtain a steady attitude when there exist uncertainties, external disturbances, actuator failures, or saturations. However, lots of nonlinear control schemes are applied on attitude control in much literature. In [3, 4], an extended state observer sliding mode control (SMC) is proposed for spacecraft attitude control with inertia uncertainty and external disturbance. In $[4,5]$, the adaptive SMC is applied to attitude stabilization with control constraints. In the above adaptive SMC scheme, it is assumed that the inertia matrix and the external disturbance are bounded and satisfy certain conditions. In [6], two quasi-continuous higherorder sliding controllers are applied to spacecraft attitudetracking. In [7], to alleviate chattering and ensure a smooth control for actuators by SMC, a second-order sliding mode controller based on anti-unwinding control method is proposed for the attitude stabilization of a rigid spacecraft. In [1], nonlinear Ho robust controller is proposed for F-16 aircraft with mass and moment inertia uncertainties. To solve the associated Hamilton-Jacobi partial differential inequality, a special Lyapunov function with mass and moment inertia uncertainties is considered. A robust control law based on the mini-max approach and the inverse optimal approach is proposed for the attitude control with external disturbances [8]. In [9], the inverse optimal feedback control based on integrator backstepping is proposed for rigid spacecraft without considering the disturbances and uncertainties. The optimal adaptive controller is designed to achieve attitude tracking for rigid spacecraft [10]. To deal with the inertia matrix uncertainty and attenuate the 
disturbance, both the adaptive control and the inverse optimal control approach are applied. However, the abovementioned controls are based on precise analytic structure of uncertainty to derive online updating algorithms for unknown but constant parameters in the system.

Actually, the inputs of spacecraft systems are usually limited, which are known as actuator saturation or input saturation. In practical systems, input saturation may lead to instability or unacceptable performance degradation for the spacecraft's attitude. To deal with the input saturation, lots of methods appeared [5, 11-15]. In [11], taking into account control input saturation, external disturbances, and parametric uncertainty, a variable structure control method is proposed for spacecraft attitude stabilization, which ensures the fast and accurate response. Thereafter, the continuous variable structure control approach is presented for attitude tracking [12]. In [13], two saturated finite-time attitude controllers based on the homogeneous method are proposed for rigid spacecraft subject to control input saturation, without considering the external disturbance and uncertainty. In [14], a saturated attitude control scheme composed of the quaternion part, the saturated angular velocity part, and the bounded antidisturbance part was proposed for spacecraft with bounded disturbances. In [15], two novel anti-unwinding attitude controllers based on the inverse optimal approach and unit quaternion are designed for spacecraft subject to uncertainty and external disturbance.

Note that the abovementioned references concentrate on the attitude control without actuator fault. In practice, besides the saturation, actuator failures also usually occur in spacecraft systems. As is known to all, control failures can lead to performance deterioration, instability, and even catastrophic accidents. Therefore, it is very important to design a controller with fault-tolerant capability to improve the spacecraft system reliability. In order to enhance the reliability of spacecraft systems, some scholars are focusing on fault-tolerant attitude control [16-19]. In [20], an adaptive fault-tolerant control method is proposed for the spacecraft subjected to two types of faults, without uncertainties and disturbances. In [16], an adaptive backstepping sliding mode control scheme is designed to achieve attitude tracking for flexible spacecraft with actuator failures, disturbances, and uncertainties. In [17], an adaptive faulttolerant control based on the fuzzy logic system and sliding mode observers is presented for near-space vehicle without the bounds of the derivative of the faults. In [18], two integral-type sliding mode control schemes are used to compensate actuator faults for spacecraft with external disturbances.

However, as far as the authors know, few papers focus on the attitude control for the rigid spacecraft subjected to uncertainties, disturbances, failures, and actuator saturation simultaneously. In [2], a nonregression-based indirect robust approach is presented to achieve attitude tracking when accounting for uncertainties, disturbances, failures, and actuator saturation simultaneously. Although the uncertainty is done by its bound, it is assumed that the external disturbance and the inertia matrix are bounded and satisfy certain conditions. In [19], a variable structure control is developed for attitude stabilization of spacecraft with partial loss of actuator effectiveness fault and actuators saturation. Then, the authors proposed an adaptive sliding mode control for spacecraft subjected to partial loss of actuator effectiveness fault, and the control law is modified to ensure that the control signal never contains saturation [21]. The neural network is used to approximate uncertainties and online updating law to estimate the bound of actuator fault without any information of the fault. In [22], based on fuzzy logic and backstepping techniques, a robust adaptive control is proposed for spacecraft in the presence of uncertainties, disturbances, actuator failures, and input saturation. Actually, the spacecraft system with uncertainties, disturbances, actuator failures, and actuator saturation simultaneously is a more complicated uncertain nonlinear system, whose typical representative is a nonaffine nonlinear system. In [23-27], the active disturbance rejection technique presented in [28] is applied to the nonaffine nonlinear system. Therefore, in this paper, a compound control based on active disturbance rejection control (ADRC) scheme and slide mode control (SMC) is designed to achieve attitude tracking for the spacecraft system. To handle the saturation, the saturation coefficient is introduced and its adaptive law is designed. The main contribution of this paper compared to others is that the proposed scheme can achieve reliability against inertia matrix uncertainties, external disturbances, actuators faults, and saturation. Moreover, the actuator faults that the scheme can deal with by the proposed scheme include partial effectiveness loss fault, additive fault, and total fault. The proposed scheme does not depend on the precise analytic structure of inertia matrix and disturbances, nor depend on their bounds, which can also be applied in spacecraft systems with time-varying parameters.

This paper is organized as follows. The spacecraft attitude tracking problem and some preliminary results are described in Section 2. The compound control scheme is proposed to achieve the attitude tracking in Section 3. Several simulation results are presented in Section 4, and conclusions are drawn in Section 5.

\section{Problem Formulation and Preliminaries}

2.1. Attitude Dynamics. The mathematical models of the rigid spacecraft attitude dynamics are given by [2-4]

$$
\begin{aligned}
\mathbf{J}(t) \dot{\omega} & =-\boldsymbol{\omega}^{\times} \mathbf{J}(t) \boldsymbol{\omega}+\mathbf{F u}+\mathbf{d}(t), \\
\dot{q}_{v} & =\frac{1}{2}\left(\mathbf{q}_{v}^{\times}+q_{0} \mathbf{I}_{3}\right) \boldsymbol{\omega} \\
\dot{q}_{0} & =-\frac{1}{2} \mathbf{q}_{v}^{T} \boldsymbol{\omega}
\end{aligned}
$$

where $\mathbf{J}(t) \in \mathbf{R}^{3 \times 3}$ is the inertia matrix of the spacecraft determined by the mass distribution of the spacecraft, and obviously, it is symmetric positive definite. $\mathbf{u} \in \mathbf{R}^{3 \times 1}$ is the actual control torque generated by thrusters. $\mathbf{F} \in \mathbf{R}^{3 \times 1}$ is the thruster distribution matrix (for a given spacecraft, $\mathbf{F}$ is available and can be adjusted by changing the locations and directions of the thrusters [3]). $\mathbf{d}(t) \in \mathbf{R}^{3 \times 1}$ is the external 
disturbance. $\mathbf{q}=\left[\mathbf{q}_{v} ; q_{0}\right] \in \mathbf{R}^{4 \times 1}$ is the unit quaternion vector representing the spacecraft's attitude orientation, satisfying $\mathbf{q}_{v}^{T} \mathbf{q}_{v}+q_{0}^{2}=1$, where $\mathbf{q}_{v}=\left[q_{1}, q_{2}, q_{3}\right]^{T}$ and $q_{0}$ are the vector part and scalar components, respectively. $\mathbf{I}_{3} \in \mathbf{R}^{3 \times 3}$ is the identity matrix, $\omega=\left[\omega_{1}, \omega_{2}, \omega_{3}\right]^{T} \in \mathbf{R}^{3}$ is the angular velocity of the spacecraft, and $\omega^{\times}$is a skew symmetric matrix of $\omega$ and has the following form:

$$
\boldsymbol{\omega}^{\times}=\left[\begin{array}{ccc}
0 & -\omega_{3} & \omega_{2} \\
\omega_{3} & 0 & -\omega_{1} \\
-\omega_{2} & \omega_{1} & 0
\end{array}\right] .
$$

Remark 1. $\mathbf{J}(t)$ may change or be uncertain over time due to fuel consumptions or onboard mission; therefore, $\mathbf{J}(t)$ should be viewed as uncertain item or unknown item so that it could not be used directly in control design. It is reasonable to assume the invertible $\mathbf{J}(t)$ in the form $\mathbf{J}(t)=\mathbf{J}_{0}+\Delta \mathbf{J}(t)$, in which $\mathbf{J}_{0}$ is the nominal inertia matrix and $\Delta \mathbf{J}(t)$ is the uncertainty. Compared to the abovementioned references, both $\mathbf{J}_{0}$ and $\Delta \mathbf{J}(t)$ are unknown here, which is the most relaxed and simple condition.

Assumption 1. q and $\omega$ are available in feedback control design. In practical, angles and angular velocities can be measured with relative sensors.

Assumption 2. The external disturbances $\mathbf{d}(t) \in \mathbf{R}^{3 \times 1}$ are bounded.

The control objective is to make the aircraft track the desired attitude dynamics when the thrusters are constrained.

The torques generated by the constrained thrusters can be described as follows:

$$
\begin{aligned}
\mathbf{u} & =\operatorname{sat}\left(\mathbf{u}_{0}\right)=\left[\operatorname{sat}\left(u_{01}\right), \ldots, \operatorname{sat}\left(u_{03}\right)\right]^{T}, \\
\operatorname{sat}\left(u_{0 i}\right) & = \begin{cases}u_{0 i}, & \left|u_{0 i}\right| \leq u_{i \max }, \quad i=1, \ldots, 3, \\
u_{i \max }, & \text { otherwise, }\end{cases}
\end{aligned}
$$

where $\mathbf{u}_{0}$ is the thruster torque without limitation. The function sat(.) is used to describe that the control torques generated by the thrusters are limited. $u_{i \max }$ indicates the maximum value of the control torque generated by the thruster.

According to $[5,29]$, the saturation function can be expressed as

$$
\mathbf{u}=\operatorname{sat}\left(\mathbf{u}_{0}\right)=\varsigma\left(\mathbf{u}_{0}\right) \mathbf{u}_{0},
$$

with

$$
\begin{aligned}
& \varsigma\left(\mathbf{u}_{0}\right)=\operatorname{diag}\left[\varsigma_{1}\left(u_{01}\right), \ldots, \varsigma_{n}\left(u_{0 n}\right)\right], \\
& \varsigma_{i}\left(u_{0 i}\right)=\left\{\begin{array}{ll}
\frac{u_{i \max }}{u_{0 i}} \operatorname{sign}\left(u_{0 i}\right), & \left|u_{0 i}\right|>u_{i \max }, \\
1, & \text { otherwise. }
\end{array} \quad i=1, \ldots, n,\right.
\end{aligned}
$$

Obviously, the coefficient $\varsigma_{i}\left(u_{0 i}\right) \in(0,1]$ denotes the saturation degree of $u_{0 i}$.

Similar to [5], the desired attitude motion is supposed to be generated by

$$
\begin{aligned}
& \dot{q}_{d v}=\frac{1}{2}\left(\mathbf{q}_{d v}^{\times}+q_{d 0} \mathbf{I}_{3}\right) \boldsymbol{\omega}_{d}, \\
& \dot{q}_{d 0}=-\frac{1}{2} \mathbf{q}_{d v}^{T} \boldsymbol{\omega}_{d},
\end{aligned}
$$

where $\mathbf{q}_{d}=\left[\mathbf{q}_{d v} ; q_{d 0}\right] \in \mathbf{R}^{4 \times 1}$ is the desired unit quaternion vector of the spacecraft, satisfying $\mathbf{q}_{d v}^{T} \mathbf{q}_{d v}+q_{d 0}^{2}=1$, where $\mathbf{q}_{d v}=\left[q_{d 1}, q_{d 2}, q_{d 3}\right]^{T}$ and $q_{d 0}$ are the vector part and scalar component, respectively. $\omega_{d}=\left[\omega_{d 1}, \omega_{d 2}, \omega_{d 3}\right]^{T} \in \mathbf{R}^{3 \times 1}$ is the desired angular velocity of the spacecraft. As in $[3,5]$, $\omega_{d}$ and $\dot{\omega}_{d}$ are assumed to be bounded.

The goal of the attitude tracking control is to design the controller so that the attitude in equations (1)-(3) tracks the desired attitude in equations (8)-(9), that is, to achieve $\mathbf{q} \longrightarrow \mathbf{q}_{d}$ and $\omega \longrightarrow \omega_{d}$. To analyse the attitude tracking problem, as in $[3,5]$, we define attitude orientation error $\mathbf{q}_{e}=\left[\mathbf{q}_{e v} ; q_{e 0}\right] \in \mathbf{R}^{4 \times 1}$ and angular velocity error $\omega_{e}=\left[\omega_{e 1}, \omega_{e 2}, \omega_{e 3}\right]^{T} \in \mathbf{R}^{3 \times 1}$ as follows:

$$
\begin{aligned}
& \mathbf{q}_{e v}=q_{d 0} \mathbf{q}_{v}-\mathbf{q}_{d v}^{\times} \mathbf{q}_{v}-q_{0} \mathbf{q}_{d v}, \\
& q_{e 0}=\mathbf{q}_{d v}^{T} \mathbf{q}_{v}+q_{0} q_{d 0}, \\
& \boldsymbol{\omega}_{e}=\boldsymbol{\omega}-\mathbf{C} \boldsymbol{\omega}_{d},
\end{aligned}
$$

where $\mathbf{C}=\left(q_{e 0}^{2}-\mathbf{q}_{e v}^{T} \mathbf{q}_{e v}\right) \mathbf{I}_{3}+2 \mathbf{q}_{e v} \mathbf{q}_{e v}^{T}-2 q_{e 0} \mathbf{q}_{e v}^{\times}$is a rotation matrix, and note that $\|\mathbf{C}\|=1$ and $C=-\omega_{e}^{\times} \mathbf{C}$.

From equations (1)-(12), the attitude orientation error $\mathbf{q}_{e}$ and angular velocity error $\omega_{e}$ can be derived as follows:

$$
\begin{aligned}
\mathbf{J}(t) \dot{\omega}_{e}= & -\boldsymbol{\omega}^{\times} \mathbf{J}(t) \boldsymbol{\omega}+\mathbf{F} \varsigma\left(\mathbf{u}_{0}\right) \mathbf{u}_{0} \\
& +\mathbf{d}(t)+\mathbf{J}(t)\left(\boldsymbol{\omega}_{e}^{\times} \mathbf{C} \boldsymbol{\omega}_{d}-\mathbf{C} \dot{\omega}_{d}\right), \\
\dot{q}_{e}= & \frac{1}{2}\left[\begin{array}{c}
\mathbf{q}_{e v}^{\times}+q_{e 0} \mathbf{I}_{3} \\
-\mathbf{q}_{e v}^{T}
\end{array}\right] \boldsymbol{\omega}_{e} .
\end{aligned}
$$

It has been proved that through equations (10)-(12), the aim of $\mathbf{q} \longrightarrow \mathbf{q}_{d}$ and $\omega \longrightarrow \omega_{d}$ would be achieved by stabilizing systems (13)-(14) in [29].

In order to stabilize systems (13)-(14), as in $[5,6]$, the sliding surface can be selected as follows:

$$
\mathbf{S}=\boldsymbol{\omega}_{e}+\mathbf{K} \mathbf{q}_{e v}
$$

where $\mathbf{K}=\operatorname{diag}\left[k_{1}, k_{2}, k_{3}\right]$ and $k_{i}>0$ is a positive-designed constant.

Differentiating equation (15) and then multiplying by $\mathbf{J}(t)$, we can obtain the following: 


$$
\dot{S}=\mathbf{J}^{-1}\left[-\boldsymbol{\omega}^{\times} \mathbf{J} \mathbf{\omega}+\mathbf{F} \varsigma\left(\mathbf{u}_{0}\right) \mathbf{u}_{0}+\mathbf{d}(t)+\mathbf{J}\left(\boldsymbol{\omega}_{e}^{\times} \mathbf{C} \mathbf{\omega}_{d}-\mathbf{C} \dot{\omega}_{d}\right)+\frac{1}{2} \mathbf{J K}\left(\mathbf{q}_{e v}^{\times}+q_{e 0} \mathbf{I}_{3}\right) \boldsymbol{\omega}_{e}\right] .
$$

In Section 3, the control $\mathbf{u}_{0}$ will be designed such that $\lim _{t \rightarrow \infty} \mathbf{S}=0$. According to Lemma 2.1 in [5], $\lim _{t \rightarrow \infty} \mathbf{S}=$ 0 can guarantee $\lim _{t \rightarrow \infty} \omega_{e}=0$ and $\lim _{t \rightarrow \infty} \mathbf{q}_{e v}=0$.

\subsection{Input to State Stability}

Lemma 1 (see [29]). Consider the system $\dot{x}=f(t, x, u)$; there exists a $C^{1}$ function $V: R_{+} \times R^{n} \longrightarrow R_{+}$so that for all $x \in R^{n}$ and $u \in R^{m}$, it satisfies

$$
\begin{aligned}
\gamma_{1}(|x|) & \leq V(t, x) \leq \gamma_{2}(|x|), \\
|x| \geq \chi(|u|) & \Longrightarrow \frac{\partial V}{\partial t}+\frac{\partial V}{\partial x} f(t, x, u) \leq-\gamma_{3}(|x|),
\end{aligned}
$$

where $\gamma_{1}, \gamma_{2}$, and xare class $\kappa_{\infty}$ functions and $\gamma_{3}$ is a class $\kappa$ function. Then, the system is input to state stable (ISS), and for all $t_{0}$ and $t$ such that $0 \leq t_{0} \leq t$, the following is satisfied:

$$
|x(t)| \leq \beta\left(\left|x\left(t_{0}\right)\right|, t-t_{0}\right)+\gamma\left(\sup _{t_{0} \leq \tau \leq t}|u(\tau)|\right)
$$

where $\beta(\cdot)$ is a class $\kappa L$ function and $\gamma=\gamma_{1}^{-1} \gamma_{2} \rho$ is a class $\kappa$ function.

\section{Compound Control for Attitude Tracking}

The first case considered here is the spacecraft with only three thrusters. Actually, there always exist two types of thruster faults in the practical spacecraft system. Therefore, partial effectiveness loss fault and additive fault are considered here. The second case considered is the spacecraft with more than three thrusters; however, some of it might experience losing power totally.

3.1. Spacecraft with Only Three Thrusters. As the spacecraft has only three thrusters, $\mathbf{F}$ in equation (1) would be $\mathbf{I}_{3}$. When partial effectiveness loss fault and additive fault happen, the attitude dynamics should be described as follows:

$$
\mathbf{J}(t) \dot{\omega}=-\boldsymbol{\omega}^{\times} \mathbf{J}(t) \boldsymbol{\omega}+\boldsymbol{\delta}(t) \mathbf{u}+\mathbf{A}(t)+\mathbf{d}(t),
$$

where $\delta(t)=\operatorname{diag}\left[\delta_{1}, \delta_{2}, \delta_{3}\right]^{T} \in \mathbf{R}^{3 \times 3} ; \delta_{i} \in(0,1]$ denotes thruster effectiveness; and when $\delta_{i}=1$ denotes that the thruster works normally, while $\delta_{i} \in(0,1)$ indicates that the thruster loses its effectiveness partially. $\mathbf{A}(t)=\operatorname{diag}\left[A_{1}(t)\right.$, $\left.A_{2}(t), A_{3}(t)\right] \in \mathbf{R}^{3 \times 3}$ indicates the additive fault, and it is bounded. by

Then, the sliding surface dynamics would be described

$$
\dot{S}=\mathbf{J}^{-1}\left[-\boldsymbol{\omega}^{\times} \mathbf{J} \boldsymbol{\omega}+\boldsymbol{\delta}(t) \mathbf{u}+\mathbf{A}(t)+\mathbf{d}(t)+\mathbf{J}\left(\boldsymbol{\omega}_{e}^{\times} \mathbf{C} \boldsymbol{\omega}_{d}-\mathbf{C} \dot{\omega}_{d}\right)+\frac{1}{2} \mathbf{J K}\left(\mathbf{q}_{e v}^{\times}+q_{e 0} \mathbf{I}_{3}\right) \boldsymbol{\omega}_{e}\right] .
$$

According to the active disturbance rejection concept in [23-26], equation (35) could be changed to the following:

$$
\dot{S}=\mathbf{G}+\mathbf{B}_{0} \varsigma\left(\mathbf{u}_{0}\right) \mathbf{u}_{0},
$$

where $\mathbf{B}_{0} \in \mathbf{R}^{3 \times 3}$ is a nonsingular matrix selected by the designer and $\mathbf{G}$ is the general uncertain item:

$$
\mathbf{G}=\mathbf{J}^{-1}\left[-\boldsymbol{\omega}^{\times} \mathbf{J} \boldsymbol{\omega}+\boldsymbol{\delta}(t) \mathbf{u}+\mathbf{A}(t)+\mathbf{d}(t)+\mathbf{J}\left(\boldsymbol{\omega}_{e}^{\times} \mathbf{C} \boldsymbol{\omega}_{d}-\mathbf{C} \dot{\omega}_{d}\right)+\frac{1}{2} \mathbf{J K}\left(\mathbf{q}_{e v}^{\times}+q_{e 0} \mathbf{I}_{3}\right) \boldsymbol{\omega}_{e}\right]-\mathbf{B}_{0} \varsigma\left(\mathbf{u}_{0}\right) \mathbf{u}_{0}
$$

It contains the unknown inertia matrix, external disturbance, and the uncertainties produced by thruster fault. To facilitate analysis, $\mathbf{B}_{0}$ is chosen as a diagonal matrix.
As in $[4,24-26]$, in order to deal with $\mathbf{G}$, the extended state observer (ESO) for equation (21) can be constructed as follows: 


$$
\begin{aligned}
\left\{\begin{array}{l}
\mathbf{E}=\mathbf{Z}_{1}-\mathbf{S}, \\
\dot{Z}_{1}=\mathbf{Z}_{2}-\beta_{1} g_{c 1}(\mathbf{E})+\mathbf{B}_{0} \varsigma\left(\mathbf{u}_{0}\right) \mathbf{u}_{0}, \\
\dot{Z}_{2}=-\beta_{2} g_{c 2}(\mathbf{E}),
\end{array}\right. \\
g_{c 1}(\mathbf{E})=\left[E_{1}, E_{2}, E_{3}\right]^{T}, \\
g_{c 2}(\mathbf{E})=\left[\begin{array}{l}
\left|E_{1}\right|^{\alpha_{1}} \operatorname{sign}\left(E_{1}\right) \\
\left|E_{2}\right|^{\alpha_{1}} \operatorname{sign}\left(E_{2}\right) \\
\left|E_{3}\right|^{\alpha_{1}} \operatorname{sign}\left(E_{3}\right)
\end{array}\right], \quad 0 \leq \alpha_{1} \leq 1,
\end{aligned}
$$

where $\mathbf{E}=\left[E_{1}, E_{2}, E_{3}\right]^{T} \in \mathbf{R}^{3 \times 1}$ is the estimation error of the ESO, $\mathbf{Z}_{1} \in \mathbf{R}^{3 \times 1}$ and $\mathbf{Z}_{2} \in \mathbf{R}^{3 \times 1}$ are the observer output, and $\beta_{1}>0, \beta_{2}>0$, and $1 / 4 \beta_{1}^{2}>\beta_{2}>\|\dot{G}\|$ are the observer gains. It has been proved in $[4,25]$ that for appropriate values of $\beta_{1}, \beta_{2}, g_{c 1}(\cdot)$, and $g_{c 2}(\cdot)$, the observer output $\mathbf{Z}_{2}$ approaches $\mathbf{G}$ and $\mathbf{Z}_{1}$ approaches $\mathbf{S}$.

With the uncertainties $\mathbf{G}$ estimated by the ESO, the sliding control law can be designed as

$$
\begin{aligned}
\mathbf{u} & =\operatorname{sat}\left(\mathbf{u}_{0}\right), \\
\mathbf{u}_{0} & =-\mathbf{B}_{0}^{-1}\left[\boldsymbol{\tau} \mathbf{S}+\boldsymbol{\sigma} \operatorname{sig}(\mathbf{S})^{r}\right]-\mathbf{B}_{0}^{-1} \hat{\rho} \frac{\mathbf{S}}{\|\mathbf{S}\|}\left(\eta+\left\|\mathbf{Z}_{2}\right\|\right) \\
\dot{\hat{\rho}} & =p_{0} \hat{\rho}^{3}\|\mathbf{S}\|\left(\eta+\left\|\mathbf{Z}_{2}\right\|\right), \quad \hat{\rho}(0)>0
\end{aligned}
$$

with $\tau=\operatorname{diag}\left[\tau_{1}, \tau_{2}, \tau_{3}\right], \tau_{i}>0, \sigma=\operatorname{diag}\left[\sigma_{1}, \sigma_{2}, \sigma_{3}\right], \sigma_{i}>0$, and $\operatorname{sig}(\mathbf{S})^{r}=\left[\left|S_{1}\right|^{r} \operatorname{sign}\left(S_{1}\right), \ldots,\left|S_{3}\right|^{r} \operatorname{sign}\left(S_{3}\right)\right]^{T}, r \in(0,1)$, where $p_{0}>0$ and $\eta>0$ are positive constant parameters chosen by the designer. $\mathbf{Z}_{2}$ is the state of ESO equation (23), which can approach $\mathbf{G}$.

Theorem 1. Consider a spacecraft system with partial effectiveness loss fault and additive fault; meanwhile, the thrusters are limited, which is stated in equation (19). If control scheme (24) is implemented, then the control objectives $\mathbf{q} \longrightarrow \mathbf{q}_{d}$ and $\omega \longrightarrow \omega_{d}$ would be achieved.

Proof. Construct the Lyapunov function as follows:

$$
V_{s}=\frac{1}{2} \mathbf{S}^{T} \mathbf{S}+\frac{1}{2 p_{0}} \tilde{\rho}^{T} \tilde{\rho},
$$

where $\widetilde{\rho}=\rho-\widehat{\rho}^{-1}$ and $p_{0}>0$ is a designed constant. In view of equation (21), its time derivative can be given as follows:

$$
\dot{V}_{s}=\mathbf{S}^{T} \dot{S}+\frac{1}{p_{0}} \tilde{\rho}^{T} \widehat{\rho}^{-2} \dot{\hat{\rho}}=\mathbf{S}^{T}\left(\mathbf{G}+\mathbf{B}_{0} \varsigma\left(\mathbf{u}_{0}\right) \mathbf{u}_{0}\right)+\frac{1}{p_{0}} \tilde{\rho}^{T} \widehat{\rho}^{-2} \dot{\hat{\rho}}
$$

Owing to both $\mathbf{B}_{0}$ and $\varsigma\left(\mathbf{u}_{0}\right)$ being diagonal matrices and combining with equation (24), equation (27) can be expressed as follows:

$$
\begin{aligned}
\dot{V}_{s}= & \mathbf{S}^{T}\left(\mathbf{G}+\varsigma\left(\mathbf{u}_{0}\right) \mathbf{B}_{0} \mathbf{u}_{0}\right)+\frac{1}{p_{0}} \tilde{\rho}^{T} \hat{\rho}^{-2} \dot{\hat{\rho}} \\
= & -\mathbf{S}^{T}\left(\varsigma\left(\mathbf{u}_{0}\right) \tau \mathbf{S}+\varsigma\left(\mathbf{u}_{0}\right) \boldsymbol{\sigma s i g n}(\mathbf{S})^{r}\right) \\
& +\mathbf{S}^{T} \mathbf{G}-\mathbf{S}^{T} \varsigma\left(\mathbf{u}_{0}\right) \hat{\rho} \frac{\mathbf{S}}{\|\mathbf{S}\|}\left(\eta+\left\|\mathbf{Z}_{2}\right\|\right)+\frac{1}{p_{0}} \tilde{\rho}^{T} \widehat{\rho}^{-2} \dot{\hat{\rho}} \\
= & -\sum_{i=1}^{3} \varsigma_{i}\left(u_{0 i}\right) \tau_{i} S_{i}^{2}-\sum_{i=1}^{3} \varsigma_{i}\left(u_{0 i}\right) \sigma_{i}\left|S_{i}\right|^{r+1} \\
& +\mathbf{S}^{T} \mathbf{G}-\frac{\hat{\rho}\left(\eta+\left\|\mathbf{Z}_{2}\right\|\right)}{\|\mathbf{S}\|} \sum_{i=1}^{3} \varsigma_{i}\left(u_{0 i}\right) S_{i}^{2}+\frac{1}{p_{0}} \tilde{\rho}^{T} \hat{\rho}^{-2} \dot{\hat{\rho}} .
\end{aligned}
$$

As in [30], since $\varsigma_{i}\left(u_{0 i}\right) \in(0,1]$, then according to the density property of the real number, there would exist a constant $\rho$ satisfying

$$
0<\rho \leq \min _{1 \leq i \leq 3}\left(\varsigma_{i}\left(u_{0 i}\right)\right) \leq 1 .
$$

According to equation (29), it yields

$$
\begin{aligned}
\dot{V}_{s} \leq & -\rho\left(\sum_{i=1}^{3} \tau_{i} S_{i}^{2}+\sum_{i=1}^{3} \sigma_{i}\left|S_{i}\right|^{r+1}\right) \\
& +\mathbf{S}^{T} \mathbf{G}-\frac{\hat{\rho}\left(\eta+\left\|\mathbf{Z}_{2}\right\|\right)}{\|\mathbf{S}\|} \sum_{i=1}^{3} \varsigma_{i}\left(u_{0 i}\right) S_{i}^{2}+\frac{1}{p_{0}} \tilde{\rho}^{T} \hat{\rho}^{-2} \dot{\hat{\rho}} .
\end{aligned}
$$

According to equation (25), it is not difficult to note that $\hat{\rho}(0)>0$ ensures that $\hat{\rho}$ is always larger than zero. Therefore, the following can be further obtained:

$$
\begin{aligned}
\dot{V}_{s} \leq & -\rho\left(\sum_{i=1}^{3} \tau_{i} S_{i}^{2}+\sum_{i=1}^{3} \sigma_{i}\left|S_{i}\right|^{r+1}\right)+\mathbf{S}^{T} \mathbf{G} \\
& -\rho \frac{\hat{\rho}\left(\eta+\left\|\mathbf{Z}_{2}\right\|\right)}{\|\mathbf{S}\|} \sum_{i=1}^{3} S_{i}^{2}+\frac{1}{p_{0}} \tilde{\rho}^{T} \hat{\rho}^{-2} \dot{\hat{\rho}} \\
\leq & -\rho\left(\sum_{i=1}^{3} \tau_{i} S_{i}^{2}+\sum_{i=1}^{3} \sigma_{i}\left|S_{i}\right|^{r+1}\right)+\mathbf{S}^{T} \mathbf{G}-\rho \hat{\rho}\left(\eta+\left\|\mathbf{Z}_{2}\right\|\right)\|\mathbf{S}\| \\
& +\frac{1}{p_{0}} \tilde{\rho}^{T} \hat{\rho}^{-2} \dot{\hat{\rho}} .
\end{aligned}
$$

According to equation (25) and $\rho=\widetilde{\rho}+\widehat{\rho}^{-1}$ in equation (31), it yields 


$$
\begin{aligned}
\dot{V}_{s} \leq & -\rho\left(\sum_{i=1}^{3} \tau_{i} S_{i}^{2}+\sum_{i=1}^{3} \sigma_{i}\left|S_{i}\right|^{r+1}\right)+\mathbf{S}^{T} \mathbf{G}-\left(\widetilde{\rho}+t \widehat{\rho}^{-1}\right) \hat{\rho}\left(\eta+\left\|\mathbf{Z}_{2}\right\|\right)\|\mathbf{S}\|+\frac{1}{p_{0}} \tilde{\rho}^{T} \widehat{\rho}^{-2} p_{0} \widehat{\rho}^{3}\|\mathbf{S}\|\left(\eta+\left\|\mathbf{Z}_{2}\right\|\right) \\
\leq & -\rho\left(\sum_{i=1}^{3} \tau_{i} S_{i}^{2}+\sum_{i=1}^{3} \sigma_{i}\left|S_{i}\right|^{r+1}\right)+\mathbf{S}^{T} \mathbf{G}-\eta\|\mathbf{S}\|-\left\|\mathbf{Z}_{2}\right\|\|\mathbf{S}\| \leq-\rho\left(\sum_{i=1}^{3} \tau_{i} S_{i}^{2}+\sum_{i=1}^{3} \sigma_{i}\left|S_{i}\right|^{r+1}\right) \\
& -\eta\|\mathbf{S}\|+\|\mathbf{S}\|\left(\|\mathbf{G}\|-\left\|\mathbf{Z}_{2}\right\|\right) \leq-\rho\left(\sum_{i=1}^{3} \tau_{i} S_{i}^{2}+\sum_{i=1}^{3} \sigma_{i}\left|S_{i}\right|^{r+1}\right)-\eta\|\mathbf{S}\|+\|\mathbf{S}\|\left\|G-\mathbf{Z}_{2}\right\| \\
\leq & -\rho \sum_{i=1}^{3} \sigma_{i}\left|S_{i}\right|^{r+1}-\eta\|\mathbf{S}\|-\rho \min _{i} \tau_{i}\|\mathbf{S}\|^{2}+\|\mathbf{S}\|\left\|\mathbf{G}-\mathbf{Z}_{2}\right\| .
\end{aligned}
$$

Then, $\left\|\mathbf{G}-\mathbf{Z}_{2}\right\|$ can be viewed as the disturbance input of the closed loop system consisting of equations (20), (23), (24), and (25). When $\|\mathbf{S}\| \geq \chi\left(\left\|\mathbf{G}-\mathbf{Z}_{2}\right\|\right)$, with $\chi\left(\left\|\mathbf{G}-\mathbf{Z}_{2}\right\|\right)=$ $1 / \rho \min _{i} \tau_{i}\left\|\mathbf{G}-\mathbf{Z}_{2}\right\|$, equation (32) is smaller than zero. Therefore, the closed loop system is ISS. Based on Lemma 1, it is easy to conclude that the estimation error $\left\|\mathbf{G}-\mathbf{Z}_{2}\right\|$ by ESO will affect that whether the dynamic of the sliding mode converges to the sliding surface $\mathbf{S}=0$. According to the principle of ESO, it is shown that $\mathbf{Z}_{2}$ can only converge into a residual set of $\mathbf{G}$, which means that $\left\|\mathbf{G}-\mathbf{Z}_{2}\right\|$ will converge into a small residual set of zero. That is to say, the dynamic of the sliding mode will be restricted to the neighborhood of the sliding surface. Fortunately, this neighborhood can be reduced to any small size by selecting the ESO parameters $\beta_{1}, \beta_{2}, g_{c 1}(\cdot)$, and $g_{c 2}(\cdot)$ and the controller parameters $\tau, \sigma$, and $\eta$. Furthermore, $\tau$ and $\sigma$ determine the speed of convergence and the final error. According to equation (24), the bigger the parameters $\tau$ and $\sigma$ are, the faster the speed converging to zero and the smaller the approach error is.

Remark 2. Obviously, $\chi\left(\left\|\mathbf{G}-\mathbf{Z}_{2}\right\|\right)=1 / \rho \min _{i} \tau_{i}\left\|\mathbf{G}-\mathbf{Z}_{2}\right\|$ is a class $\kappa_{\infty}$ function, so Lyapunov function (26) satisfies Lemma 1; that is to say, the closed loop system is ISS.

Remark 3. In order to ensure the stability for the dynamic of the sliding mode and the ability to approach the selected sliding surface, $\hat{\rho}(0)$ and $\tau_{i}$ should satisfy $\hat{\rho}(0) \tau_{i}>1$. The reason is that $\dot{V}_{s} \leq-\rho \min _{i} \tau_{i}\|\mathbf{S}\|^{2}+\|\mathbf{S}\|^{2}+\left\|\mathbf{G}-\mathbf{Z}_{2}\right\|^{2} / 4$, and $\rho$ is designed as an incremental function.

Remark 4. ${ }^{B_{0}}$ determines the effectiveness of the active disturbance rejection. Although ${ }^{B_{0}}$ can be selected as an arbitrary diagonal matrix, the closer ${ }^{B_{0}}$ is to the real value, the better the effect of the active disturbance rejection.

Remark 5. As $\mathbf{S}$ crosses the zero, the controller in equation (24) will be discontinuous, which will result in chattering. Therefore, the bounded layer should be introduced to eliminate the chattering. As in [5], equation (24) can be modified as follows:

$$
\mathbf{u}_{0}=-\mathbf{B}_{0}^{-1}\left[\boldsymbol{\tau} \mathbf{S}+\boldsymbol{\sigma} \operatorname{sig}(\mathbf{S})^{r}\right]-\mathbf{B}_{0}^{-1} \widehat{\rho} \frac{\mathbf{S}}{\|\mathbf{S}\|+\varepsilon}\left(\eta+\left\|\mathbf{Z}_{2}\right\|\right)
$$

where $\varepsilon>0$ is the bounded layer, and it should be selected to be small enough.

3.2. Spacecraft with More than Three Thrusters. When the spacecraft has more than three thrusters, some of which might suffer from total fault, then the attitude dynamics would be described as follows [2]:

$$
\mathbf{J}(t) \dot{\omega}=-\boldsymbol{\omega}^{\times} \mathbf{J}(t) \boldsymbol{\omega}+\mathbf{F} \Gamma \mathbf{u}+\mathbf{d}(t),
$$

where $\Gamma=\operatorname{diag}\left[\Gamma_{1}, \ldots, \Gamma_{n}\right] \in \mathbf{R}^{n \times n}$ denotes the work status of the thrusters. $\Gamma_{i}=0$ means that the thruster has either totally failed or been shut down purposely.

Correspondingly, sliding surface dynamics (16) would be described by

$$
\begin{aligned}
\dot{S}= & \mathbf{J}^{-1}\left[-\boldsymbol{\omega}^{\times} \mathbf{J} \boldsymbol{\omega}+\mathbf{F} \Gamma \mathbf{u}+\mathbf{d}(t)+\mathbf{J}\left(\boldsymbol{\omega}_{e}^{\times} \mathbf{C} \boldsymbol{\omega}_{d}-\mathbf{C} \dot{\omega}_{d}\right)\right. \\
& \left.+\frac{1}{2} \mathbf{J K}\left(\mathbf{q}_{e v}^{\times}+q_{e 0} \mathbf{I}_{3}\right) \boldsymbol{\omega}_{e}\right] .
\end{aligned}
$$

Firstly, we can choose $\mathbf{B}_{0}$ as $\mathbf{B}_{0}=\left[\begin{array}{llllll}1 & 0 & 0 & 0 & \ldots & 0 \\ 0 & 1 & 0 & 0 & \ldots & 0 \\ 0 & 0 & 1 & 0 & \ldots & 0\end{array}\right]_{3 \times n}$. According to the active disturbance rejection concept in [23-26], equation (35) could be changed to the following:

$$
\dot{S}=\mathbf{G}+\mathbf{B}_{0} \varsigma\left(\mathbf{u}_{0}\right) \mathbf{u}_{0},
$$

where $\mathbf{G}$ is the total uncertain item, which can be written as follows:

$$
\begin{aligned}
\mathbf{G}= & \mathbf{J}^{-1}\left[-\boldsymbol{\omega}^{\times} \mathbf{J} \boldsymbol{\omega}+\mathbf{F} \Gamma \mathbf{u}+\mathbf{d}(t)+\mathbf{J}\left(\boldsymbol{\omega}_{e}^{\times} \mathbf{C} \boldsymbol{\omega}_{d}-\mathbf{C} \dot{\omega}_{d}\right)\right. \\
& \left.+\frac{1}{2} \mathbf{J K}\left(\mathbf{q}_{e v}^{\times}+q_{e 0} \mathbf{I}_{3}\right) \boldsymbol{\omega}_{e}\right]-\mathbf{B}_{0} \varsigma\left(\mathbf{u}_{0}\right) \mathbf{u}_{0} .
\end{aligned}
$$

It contains the unknown inertia matrix, external disturbance, and uncertainties produced by thrusters fault.

And then, it is interesting to find that the sliding dynamics can be stabilized with a slight modification to the above controller, as follows: 


$$
\begin{aligned}
& \mathbf{u}=\operatorname{sat}\left(\mathbf{u}_{0}\right), \\
& \mathbf{u}_{0}=-\mathbf{B}_{0}^{T}\left[\boldsymbol{\tau} \mathbf{S}+\boldsymbol{\sigma} \operatorname{sig}(\mathbf{S})^{r}\right]-\mathbf{B}_{0}^{T} \widehat{\rho} \frac{\mathbf{S}}{\|\mathbf{S}\|}\left(\eta+\left\|\mathbf{Z}_{2}\right\|\right), \\
& \dot{\hat{\rho}}=p_{0} \hat{\rho}^{3}\|\mathbf{S}\|\left(\eta+\left\|\mathbf{Z}_{2}\right\|\right), \quad \hat{\rho}(0)>0,
\end{aligned}
$$

where all the control parameters are defined as before. The stability of the closed loop system can be analyzed by using the same Lyapunov function. Combining controller (38) and adaptive law (39), the derivative of the Lyapunov function can be given as follows:

$$
\begin{aligned}
\dot{V}_{s}= & \mathbf{S}^{T} \mathbf{G}-\mathbf{S}^{T} \mathbf{B}_{0} \varsigma\left(\mathbf{u}_{0}\right) \mathbf{B}_{0}^{T}\left[\tau \mathbf{S}+\boldsymbol{\sigma s i g}(\mathbf{S})^{r}\right] \\
& -\mathbf{S}^{T} \mathbf{B}_{0} \varsigma\left(\mathbf{u}_{0}\right) \mathbf{B}_{0}^{T} \widehat{\rho} \frac{\mathbf{S}}{\|\mathbf{S}\|}\left(\eta+\left\|\mathbf{Z}_{2}\right\|\right) \\
& +\frac{1}{p_{0}} \tilde{\rho}^{T} \widehat{\rho}^{-2} p_{0} \hat{\rho}^{3}\|\mathbf{S}\|\left(\eta+\left\|\mathbf{Z}_{2}\right\|\right) .
\end{aligned}
$$

Note that the multiplication of a vector and a diagonal matrix in equation (40) satisfies the following:

$$
\mathbf{S}^{T} \mathbf{B}_{0} \varsigma\left(\mathbf{u}_{0}\right)=\bar{\varsigma} \operatorname{diag}\left(\mathbf{S}^{T} \mathbf{B}_{0}\right) \text { with } \bar{\varsigma}=\left[\varsigma_{1}, \ldots, \varsigma_{n}\right] .
$$

Then, it is not difficult to calculate the right side of equation (40) and it can be expressed as

$$
\dot{V}_{s}=-\sum_{i=1}^{3} \varsigma_{i}\left(u_{0 i}\right) \tau_{i} S_{i}^{2}-\sum_{i=1}^{3} \varsigma_{i}\left(u_{0 i}\right) \sigma_{i}\left|S_{i}\right|^{r+1}+\mathbf{S}^{T} \mathbf{G}-\frac{\hat{\rho}\left(\eta+\left\|\mathbf{Z}_{2}\right\|\right)}{\|\mathbf{S}\|} \sum_{i=1}^{3} \varsigma_{i}\left(u_{0 i}\right) S_{i}^{2}+\tilde{\rho}^{T} \widehat{\rho}\|\mathbf{S}\|\left(\eta+\left\|\mathbf{Z}_{2}\right\|\right)
$$

According to equation (39), $\hat{\rho}$ is a positive parameter. Combining equation (29), we can obtain the following:

$$
\begin{gathered}
\dot{V}_{s} \leq-\rho \sum_{i=1}^{3} \tau_{i} S_{i}^{2}-\rho \sum_{i=1}^{3} \sigma_{i}\left|S_{i}\right|^{r+1}+\mathbf{S}^{T} \mathbf{G}-\frac{\hat{\rho}\left(\eta+\left\|\mathbf{Z}_{2}\right\|\right)}{\|\mathbf{S}\|} \rho \sum_{i=1}^{3} S_{i}^{2}+\tilde{\rho}^{T} \hat{\rho}\|\mathbf{S}\|\left(\eta+\left\|\mathbf{Z}_{2}\right\|\right) \leq-\rho \sum_{i=1}^{3} \tau_{i} S_{i}^{2}-\rho \sum_{i=1}^{3} \sigma_{i}\left|S_{i}\right|^{r+1}+\mathbf{S}^{T} \mathbf{G} \\
-\frac{\hat{\rho}\left(\eta+\left\|\mathbf{Z}_{2}\right\|\right)}{\|\mathbf{S}\|}\left(\widetilde{\rho}+t \hat{\rho}^{-1}\right) \sum_{i=1}^{3} S_{i}^{2}+\tilde{\rho}^{T} \hat{\rho}\|\mathbf{S}\|\left(\eta+\left\|\mathbf{Z}_{2}\right\|\right) \leq-\rho \sum_{i=1}^{3} \tau_{i} S_{i}^{2}-\rho \sum_{i=1}^{3} \sigma_{i}\left|S_{i}\right|^{r+1}+\|\mathbf{S}\|\left(\|\mathbf{G}\|-\left\|\mathbf{Z}_{2}\right\|\right) .
\end{gathered}
$$

Consider $\left\|\mathbf{G}-\mathbf{Z}_{2}\right\|$ as the disturbance input of the closed loop system consisting of equations (35), (23), (38), and (39). When $\|\mathbf{S}\| \geq \chi\left(\left\|\mathbf{G}-\mathbf{Z}_{2}\right\|\right)$, with $\chi\left(\left\|\mathbf{G}-\mathbf{Z}_{2}\right\|\right)=1 / \rho \min _{i} \tau_{i} \| \mathbf{G}-$ $\mathbf{Z}_{2} \|$, equation (43) is less than zero. Therefore, the entire closed-loop system is ISS for $\left\|\mathbf{G}-\mathbf{Z}_{2}\right\|$, and the following theorem can be established.

Theorem 2. Consider a spacecraft system; meanwhile, the thrusters are limited, which is stated in equation (34). If control scheme (38) is implemented, then the control objectives $\mathbf{q} \longrightarrow \mathbf{q}_{d}$ and $\omega \longrightarrow \omega_{d}$ would be achieved.

Remark 6. Similar to Remark 5, in order to eliminate the chattering, equation (38) can be modified as follows:

$$
\mathbf{u}_{0}=-\mathbf{B}_{0}^{T}\left[\mathbf{\tau} \mathbf{S}+\boldsymbol{\sigma} \operatorname{sig}(\mathbf{S})^{r}\right]-\mathbf{B}_{0}^{T} \widehat{\rho} \frac{\mathbf{S}}{\|\mathbf{S}\|+\varepsilon}\left(\eta+\left\|\mathbf{Z}_{2}\right\|\right),
$$

where $\varepsilon>0$ is the bounded layer and it should be selected to be small enough.

\section{Simulation Results}

To verify the effect of the scheme proposed above, several simulations on a spacecraft under various conditions are conducted. The limit of thrusters is selected as $u_{i \max } \leq 5 \mathrm{~N} \cdot \mathrm{m}$. The initial values for the spacecraft are selected as $\mathbf{q}(0)=[0.3,-0.2,-0.3,0.8832]^{T}$ and $\omega(0)=$ $[0,0,0]^{T} \mathrm{rad} / \mathrm{s}$. The initial values for the desired unit quaternion is set to $\mathbf{q}_{d}(0)=[0,0,0,1]^{T}$. The unknown nominal inertial matrix and its uncertainties are chosen as

$$
\mathbf{J}_{0}=\left[\begin{array}{ccc}
20 & 1.2 & 0.9 \\
1.2 & 17 & 0.4 \\
0.9 & 1.4 & 15
\end{array}\right] \mathrm{kg} \cdot \mathrm{m}^{2},
$$

$$
\Delta \mathbf{J}=\operatorname{diag}[\sin (0.1 t), 2 \sin (0.2 t), 3 \sin (0.3 t)] \mathrm{kg} \cdot \mathrm{m}^{2},
$$

and the disturbances are selected as $\mathbf{d}(t)=\left[\begin{array}{l}0.1 \sin (0.1 t) \\ 0.2 \sin (0.2 t) \\ 0.3 \sin (0.3 t)\end{array}\right] \mathrm{N} \cdot \mathrm{m}$. 
The ESO parameters are designed as $\beta_{1}=70, \beta_{2}=30$ and $\alpha_{1}=0.25$. And, the initial states of ESO are set to $\mathbf{Z}_{1}(0)=\mathbf{Z}_{2}(0)=\left[\begin{array}{lll}0 & 0 & 0\end{array}\right]^{T}$. The parameters of the control law in equations (24), (25), (38), and (39) are selected as $\mathbf{K}=2 \mathbf{I}_{3}, \tau=50 \mathbf{I}_{3}, \sigma=0.01 \mathbf{I}_{3}, r=0.3, p_{0}=1, \eta=20$, and $\varepsilon=0.1$. The initial value for adaptive law is set to $\widehat{\rho}(0)=0.1$.

In order to verify the performance of ESO, the desired angular velocity is set to a sinusoidal signal, such as $\omega_{d}(t)=$ $[0.5 \sin (\pi t / 100), 0.5 \sin (2 \pi t / 100), 0.5 \sin (3 \pi t / 100)] \mathrm{rad} / \mathrm{s}$.

4.1. Simulation for Spacecraft with Only Three Thrusters. In this section, it is assumed that thrusters have partial effectiveness loss fault and additive fault, such as $\delta_{i}(t)=$ $0.8+0.1 \sin (t)$ and $A_{i}(t)=10+10 \sin (t)$.

Simulation results are given to show the performance of control law (24). Figure 1 demonstrates the attitude quaternion tracking error. The attitude quaternion tracking error curve indicates that the proposed control law achieves the desired attitude tracking in $5 \mathrm{~s}$. The spacecraft has achieved the desired angular velocity in $5 \mathrm{~s}$ from the angular tracking error curve exhibited in Figure 2. From Figures 1 and 2, the validity of the conclusion of Theorem 1 can be verified such that control law (24) can make the spacecraft converge to the desired attitude and angular velocity in spite of the saturation, uncertainties, and disturbances.

From the sliding surface depicted in Figure 3, it could be drawn that the dynamic of sliding mode converge into the neighborhood of the sliding surface $\mathbf{S}=0$ very quickly. According to equation (15), as $\mathbf{S}$ is equal to $0, \omega_{e}=-\mathbf{K} \mathbf{q}_{e v}$, which is exactly reflected in Figures 1 and 2 . The control torques produced by three thrusters are illustrated in Figure 4 . They are limited by $u_{i \max }=5 \mathrm{~N} \cdot \mathrm{m}$. The adaptive law depicted in Figure 5 shows that although the parameter $\widehat{\rho}$ cannot converge to a certain constant, its change is very slow. However, the dynamic of the sliding mode just only converges into the neighborhood of the sliding surface $\mathbf{S}=0$ and not converges to $\mathbf{S}=0$. The performance of ESO is illustrated in Figure 6, which verifies that the total influence produced by the absolutely unknown inertial matrix, external disturbance, and actuator fault can be well estimated via ESO.

4.2. Simulation for Spacecraft with More than Three Thrusters. In this section, three thrusters with fading fault and the other three with totally failed fault are assumed, which is depicted as follows [2]:

$$
\Gamma=\operatorname{diag}[0.5,0.7,0.7,0,0,0] \in \mathbf{R}^{n \times n} .
$$

Assume that the six thrusters are distributed as

$$
\mathbf{F}=\left[\begin{array}{cccccc}
1 & 0 & 0 & 0.2 & 0.1 & 0.3 \\
0 & 1 & 0 & 0.6 & 0.5 & 0.3 \\
0 & 0 & 1 & 0 & 0.2 & 0.4
\end{array}\right]
$$

For the simulation purposes, the system's initial states, inertial matrix and its uncertainties, disturbances, and other parameters are all the same as above.

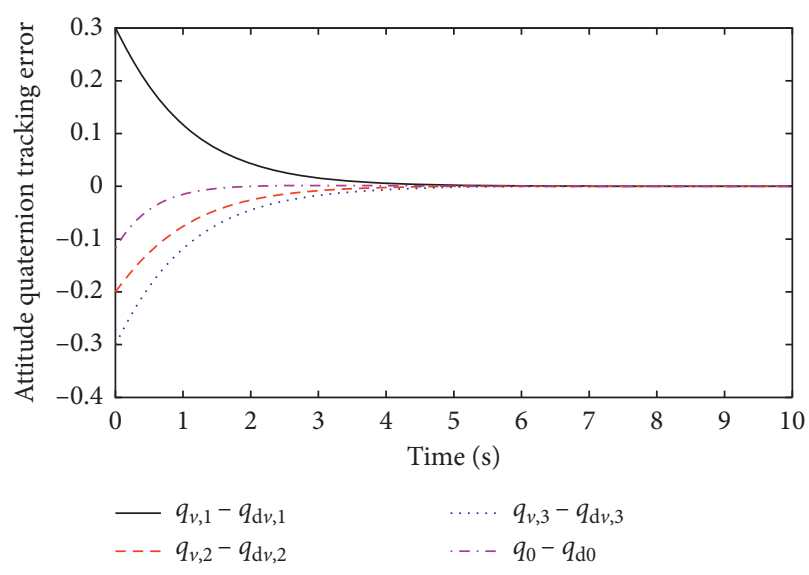

FIGURE 1: Attitude quaternion tracking error.

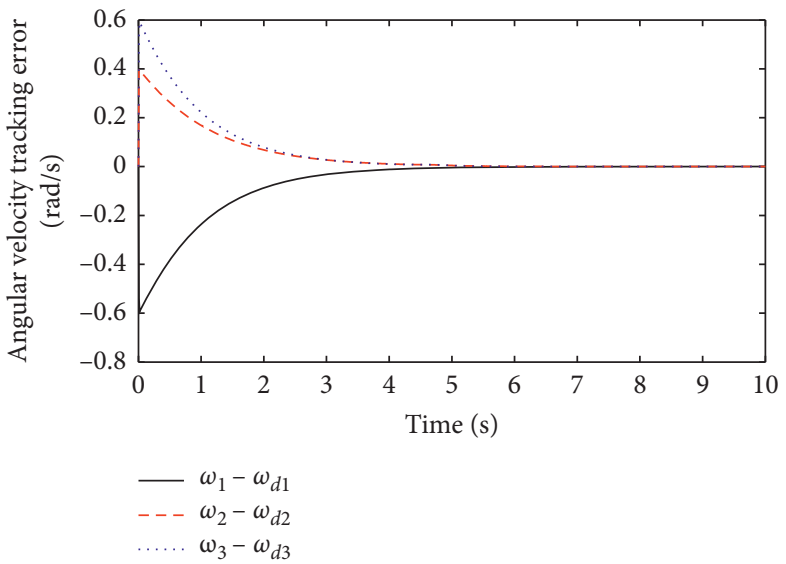

FIGURE 2: Angular velocity tracking error.

Figure 7 demonstrates the attitude quaternion tracking error. The attitude quaternion tracking error curve indicates that the proposed control law achieves the desired attitude tracking in $5 \mathrm{~s}$. The spacecraft has achieved the desired angular velocity in $5 \mathrm{~s}$ from the angular tracking error curve exhibited in Figure 8. From Figures 7 and 8, the validity of the conclusion of Theorem 2 could be verified such that control law (38) can make the spacecraft converge to the desired attitude and angular velocity in spite of the saturation, uncertainties, and disturbances.

From the sliding surface depicted in Figure 9, it could be drawn that the dynamic of sliding mode converge into the neighborhood of the sliding surface $\mathbf{S}=0$ very quickly. According to equation (15), as $\mathbf{S}$ is equal to $0, \omega_{e}=-\mathbf{K} \mathbf{q}_{e v}$, which is exactly reflected in Figures 7 and 8. The control torques produced by six thrusters are illustrated in Figure 10. They are limited by $u_{i \max }=5 \mathrm{~N} \cdot \mathrm{m}$. From Figure 10 , it is obviously deduced that three thrusters show totally failed fault which is consistent with equation (46).

Define $\mathbf{T}=\mathbf{F} \Gamma \varsigma(\mathbf{u}) \mathbf{u}$; then, the control torques for spacecraft are illustrated in Figure 11. It can be seen that the applied moment for spacecraft does not exceed the upper limit. The adaptive law depicted in Figure 12 shows that although the parameter $\hat{\rho}$ cannot converge to a certain 


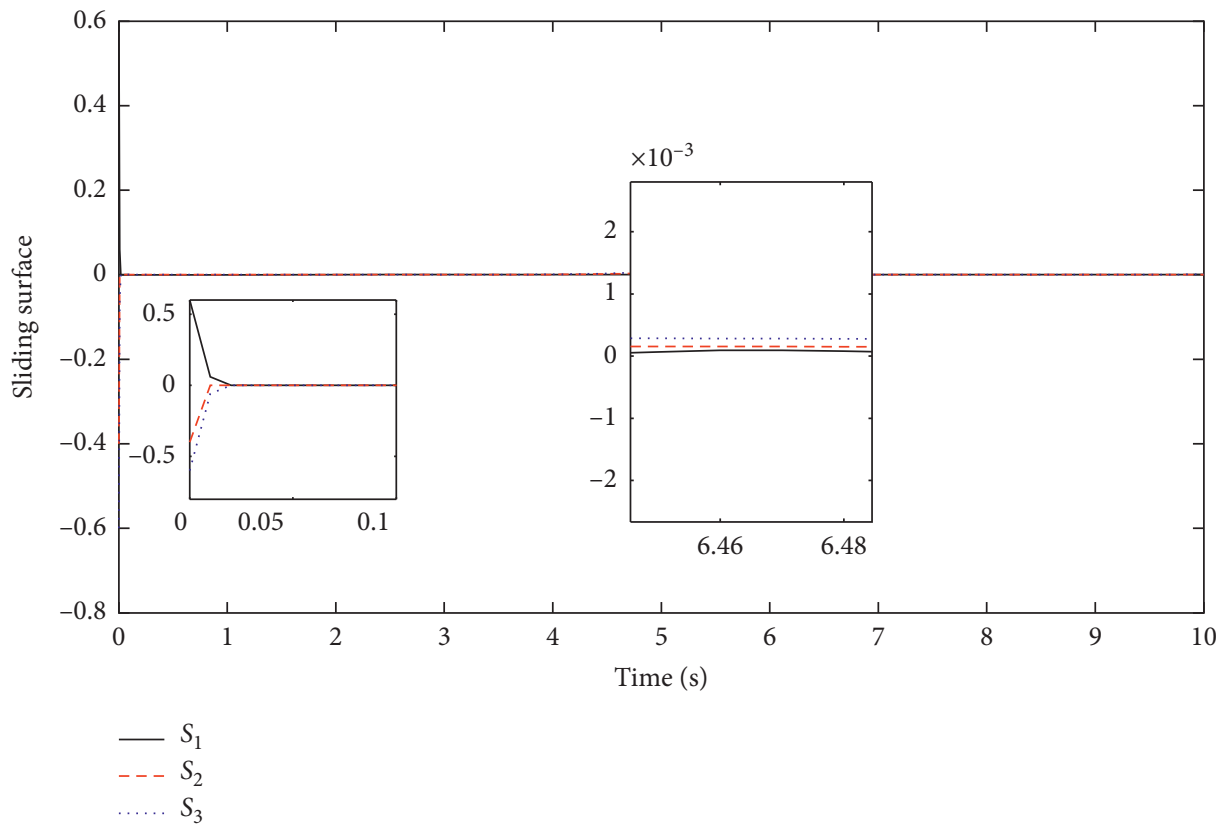

FIgURE 3: Sliding surface.

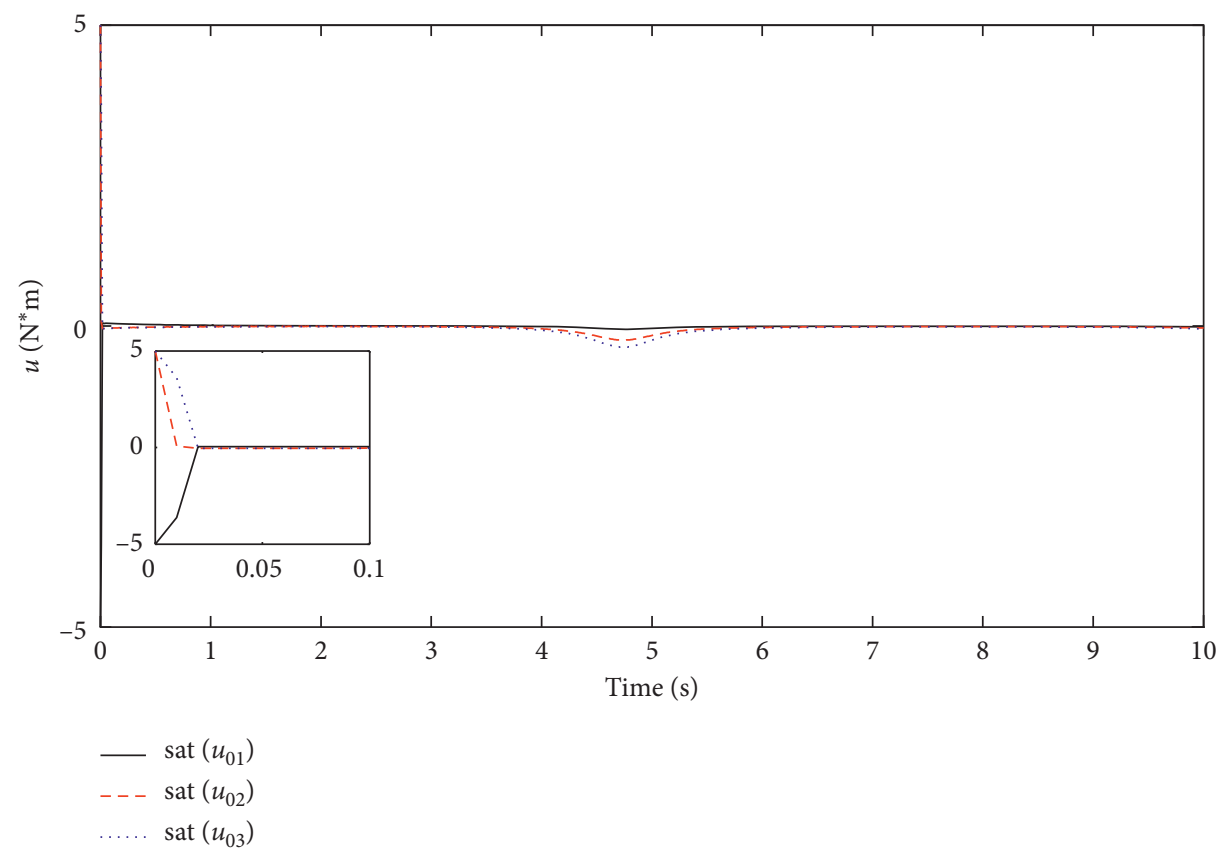

FIgUre 4: Control input. 


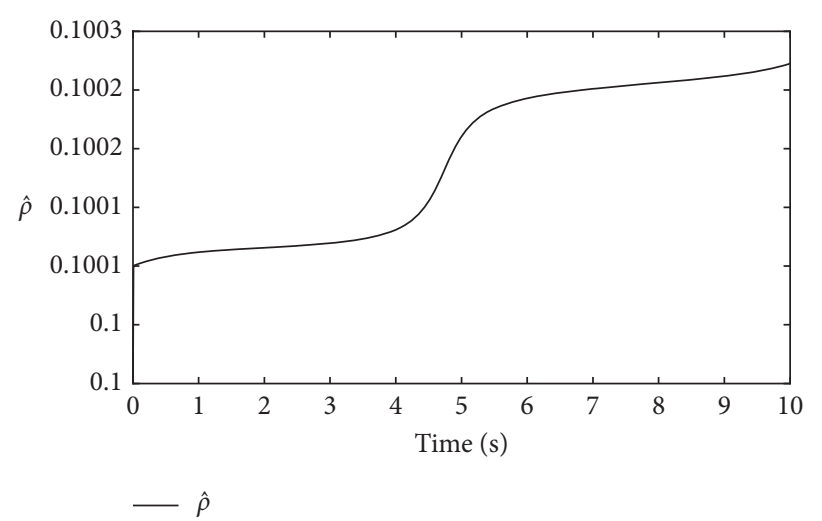

FIgURE 5: Adaptive law of the parameter $\hat{\rho}$.
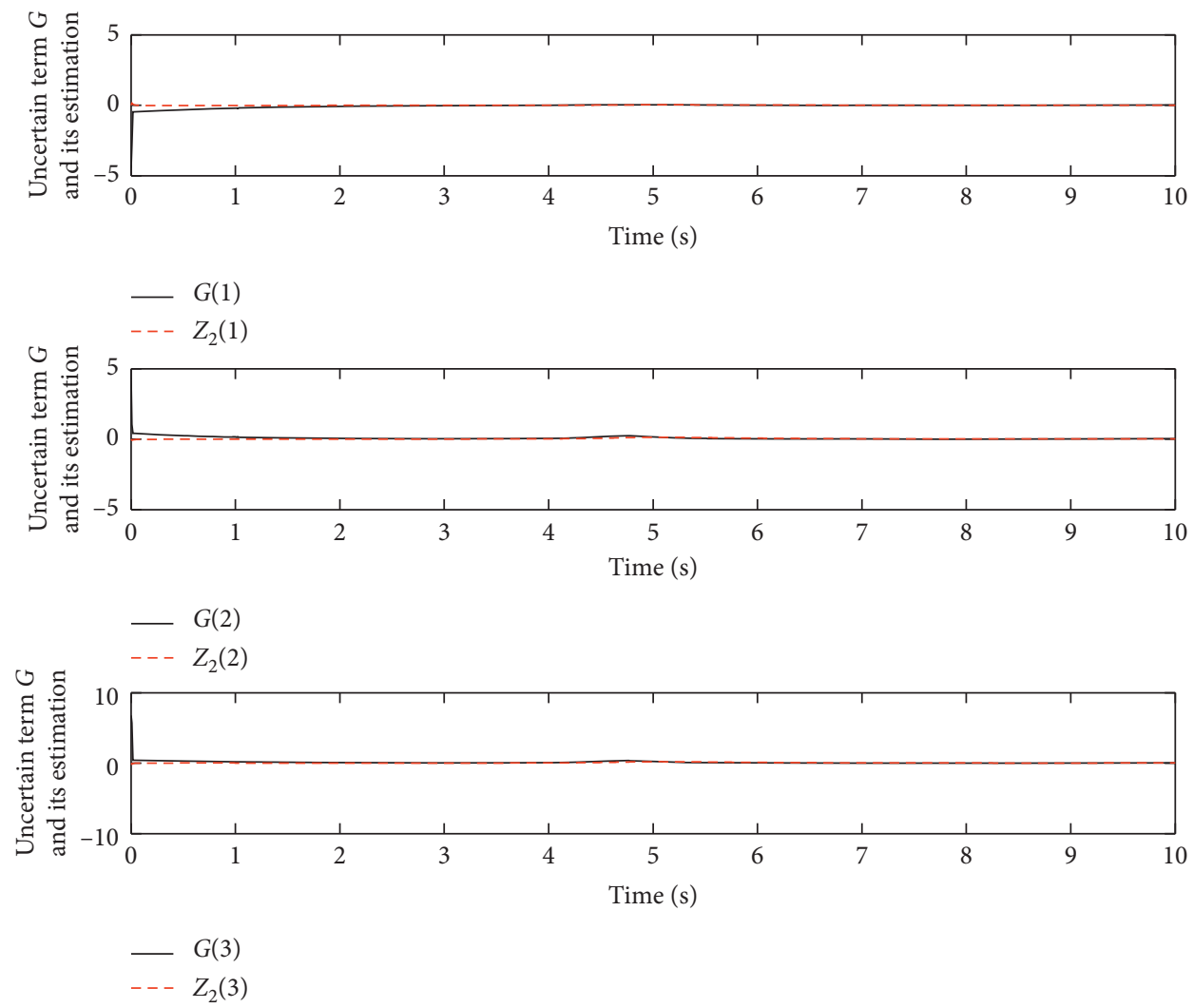

Figure 6: Estimation of general uncertainties by ESO.

constant, its change is very slow. However, the dynamic of sliding mode just only converges into the neighborhood of the sliding surface $\mathbf{S}=0$ and not converges to $\mathbf{S}=0$. The performance of ESO is illustrated in Figure 13, which verifies that the total influence produced by the absolutely unknown inertial matrix, external disturbance, and actuator fault can be well estimated via ESO.

Based on Figures 1-13, it can be concluded that the proposed scheme has achieved a good performance on attitude tracking by using ADRC and SMC. 


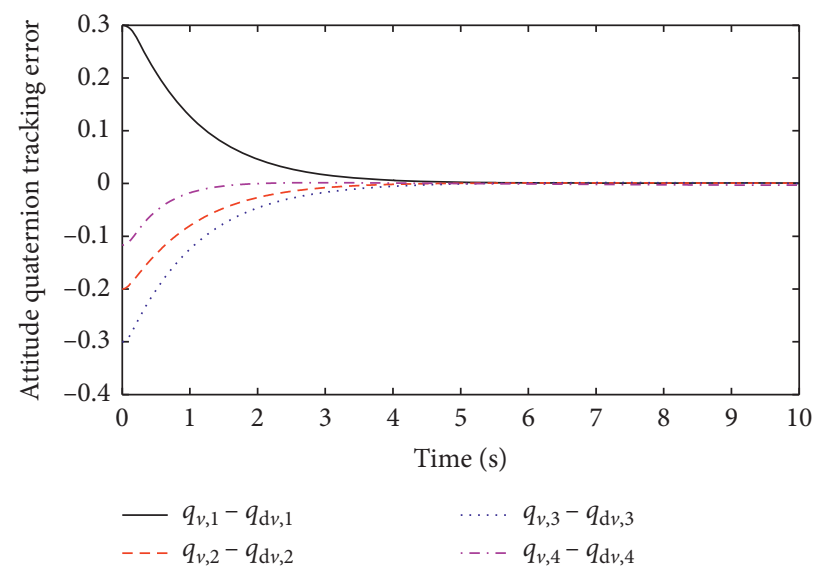

Figure 7: Attitude quaternion tracking error.

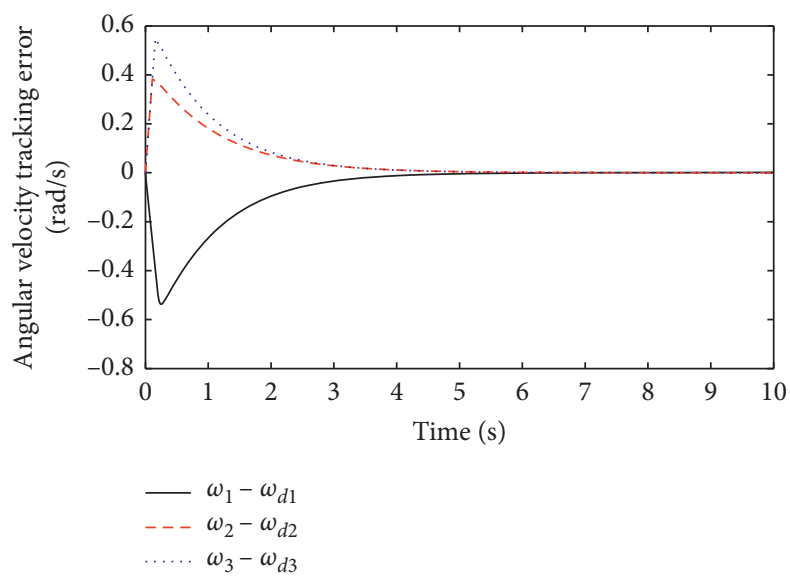

Figure 8: Angular velocity tracking error.

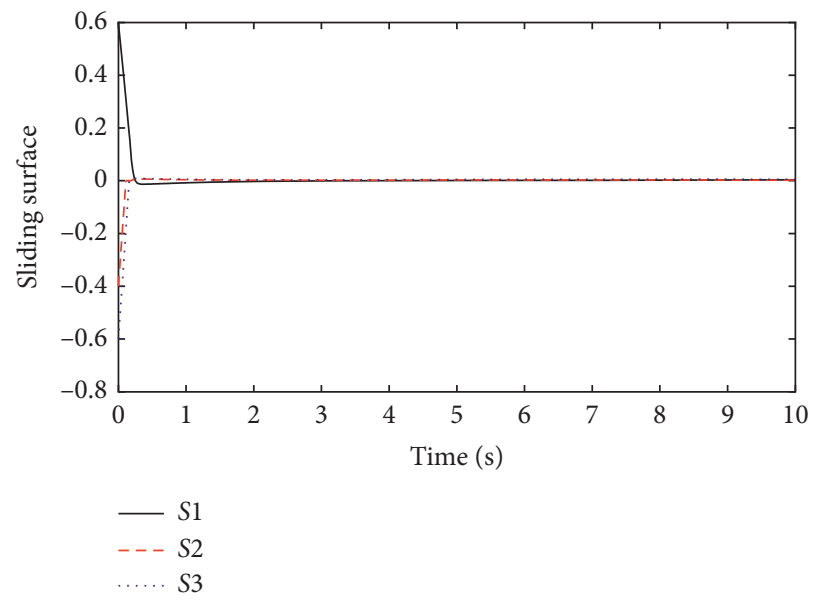

FIGURE 9: Sliding surface. 

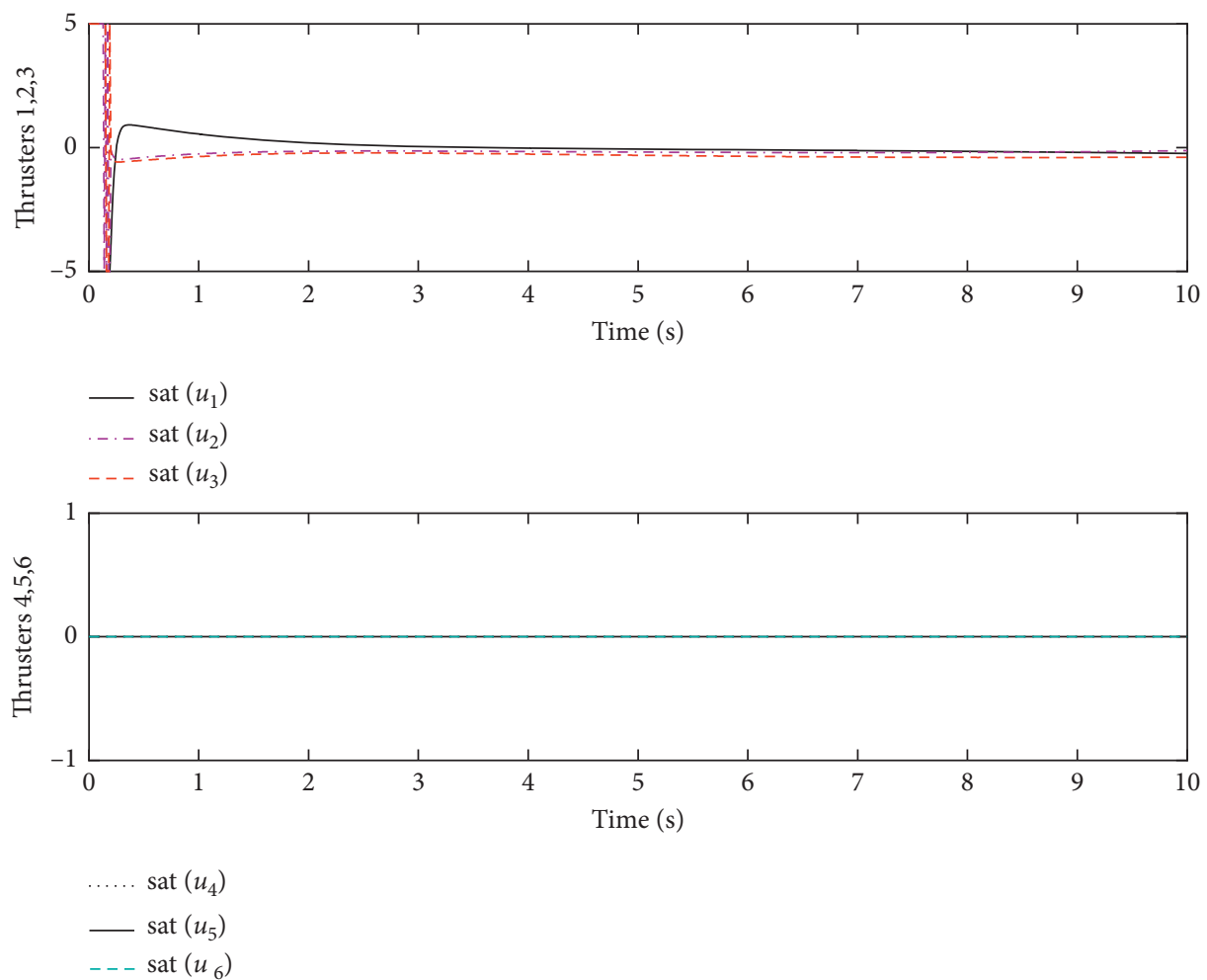

Figure 10: Control input produced by six thrusters.

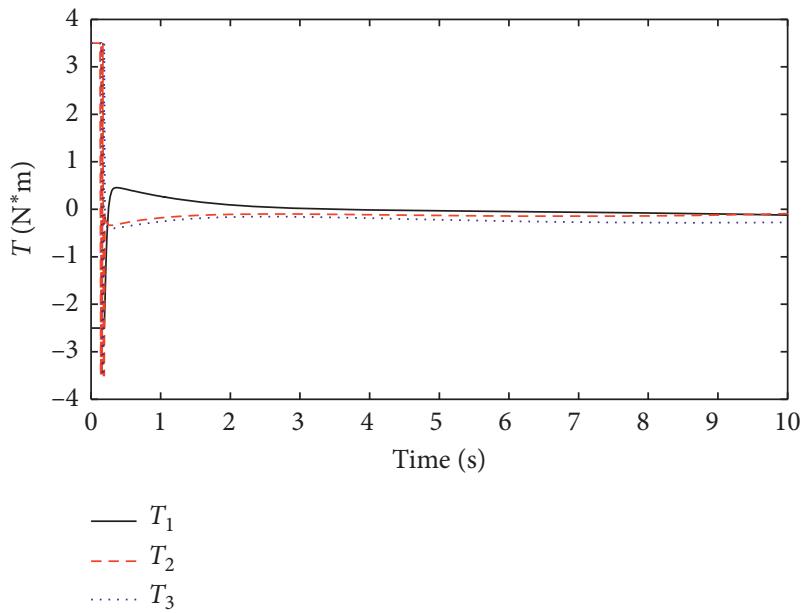

FIgUre 11: Control inputs acting on aircraft. 


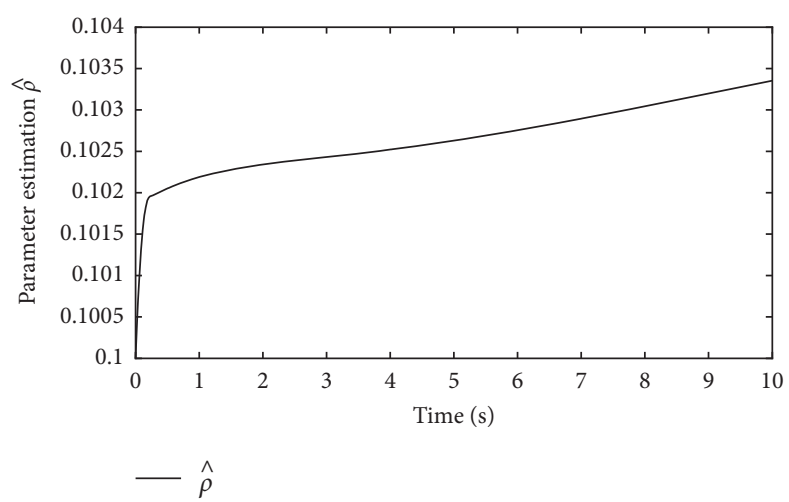

Figure 12: Adaptive law of the parameter $\hat{\rho}$.
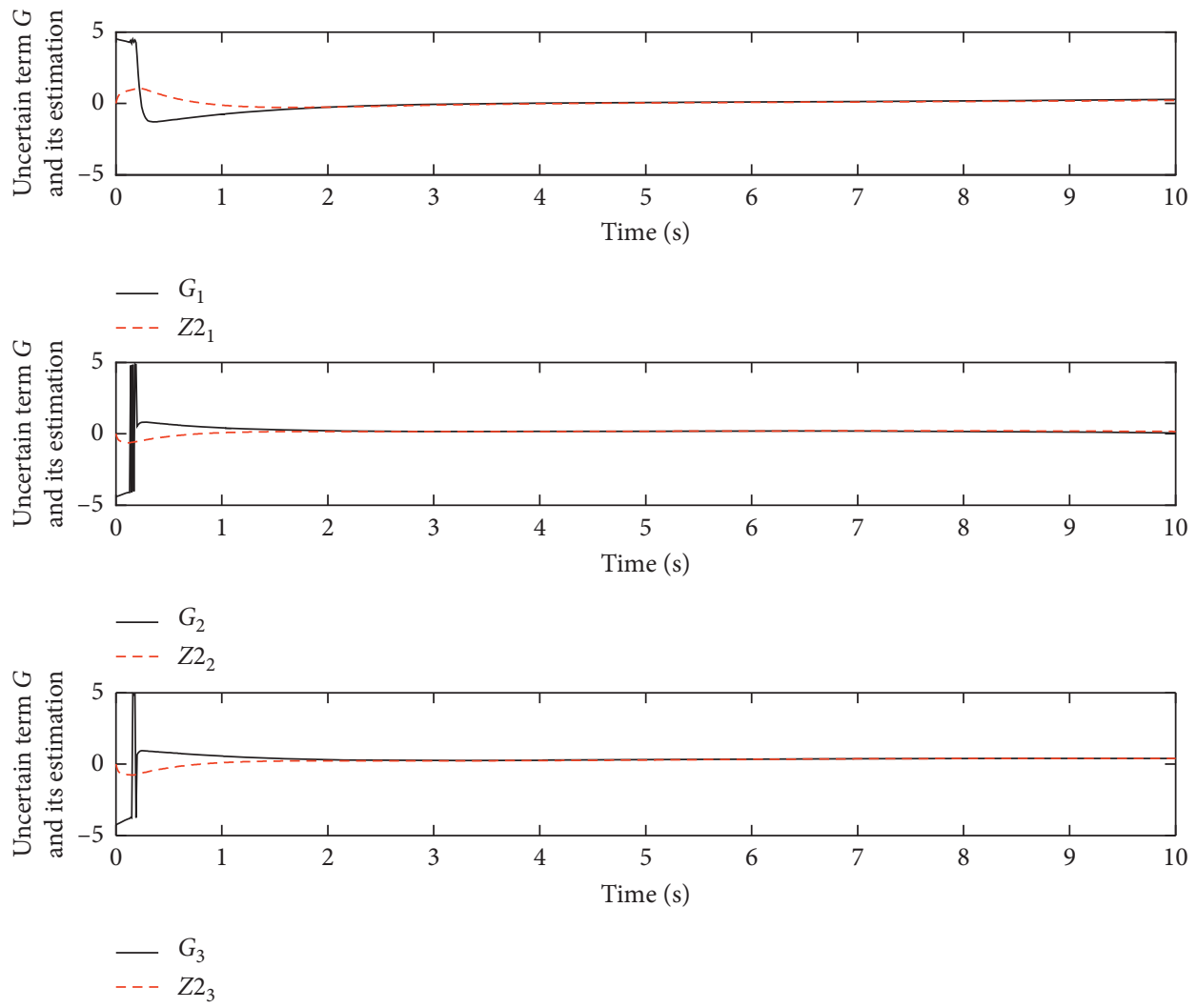

Figure 13: Estimation of general uncertainties by ESO.

\section{Conclusions}

In this paper, the attitude tracking problem for a spacecraft with uncertainties, external disturbances, actuator failures, and saturations is considered. The developed scheme for attitude tracking does not need to know the structure or upper bound information of the inertial matrix uncertainties and external disturbances. Based on ADR concept, the total uncertainties are attenuated by ESO, and the parameter adaptive method is used to deal with the thruster saturation problem; meanwhile, the sliding mode control law ensures the attitude tracking effect. Moreover, the proposed method can be well accommodated for the spacecraft with part of thrusters with total fault and saturation. Several simulation results have proved the effect of the proposed method.

\section{Data Availability}

The data used to support the findings of this study are included within the article.

\section{Conflicts of Interest}

The authors declare that they have no conflicts of interest. 


\section{Acknowledgments}

This work was supported in part by the Natural Science Foundation of Shandong Province, China (Grant no. Zr2019mf065).

\section{References}

[1] C.-C. Kung, "Nonlinear Ho robust control applied to F-16 aircraft with mass uncertainty using control surface inverse algorithm," Journal of the Franklin Institute, vol. 345, no. 8, pp. 851-876, 2008.

[2] W. Cai, X. H. Liao, and Y. D. Song, "Indirect robust adaptive fault-tolerant control for attitude tracking of spacecraft," Journal of Guidance, Control, and Dynamics, vol. 31, no. 5, pp. 1456-1463, 2008.

[3] Z. Zhu, Y. Xia, M. Fu, and S. Wang, "Attitude tracking of rigid spacecraft based on extended state observer," in Proceedings of the 20103 rd International Symposium on Systems and Control in Aeronautics and Astronautics, pp. 621-626, IEEE, Harbin, China, June 2010.

[4] Y. Xia, Z. Zhu, M. Fu, and S. Wang, "Attitude tracking of rigid spacecraft with bounded disturbances," IEEE Transactions on Industrial Electronics, vol. 58, no. 2, pp. 647-659, 2011.

[5] Z. Zhu, Y. Xia, and M. Fu, "Adaptive sliding mode control for attitude stabilization with actuator saturation," IEEE Transactions on Industrial Electronics, vol. 58, no. 10, pp. 48984907, 2011.

[6] C. Pukdeboon, A. S. I. Zinober, and M.-W. L. Thein, "Quasicontinuous higher order sliding-mode controllers for spacecraft-attitude-tracking maneuvers," IEEE Transactions on Industrial Electronics, vol. 57, no. 4, pp. 1436-1444, 2010.

[7] P. M. Tiwari, S. Janardhanan, and M. un-Nabi, "Spacecraft anti-unwinding attitude control using second-order sliding mode," Asian Journal of Control, vol. 20, no. 1, pp. 455-468, 2017.

[8] Y. Park, "Robust and optimal attitude stabilization of spacecraft with external disturbances," Aerospace Science and Technology, vol. 9, no. 3, pp. 253-259, 2005.

[9] M. Krstic and P. Tsiotras, "Inverse optimal stabilization of a rigid spacecraft," IEEE Transactions on Automatic Control, vol. 44, no. 5, pp. 1042-1049, 1999.

[10] W. Luo, Y.-C. Chu, and K.-V. Ling, "Inverse optimal adaptive control for attitude tracking of spacecraft," IEEE Transactions on Automatic Control, vol. 50, no. 11, pp. 1639-1654, 2005.

[11] J. D. Boskovic, S.-M. Li, and R. K. Mehra, "Robust adaptive variable structure control of spacecraft under control input saturation," Journal of Guidance, Control, and Dynamics, vol. 24, no. 1, pp. 14-22, 2001.

[12] J. D. Boskovic, S.-M. Li, and R. K. Mehra, "Robust tracking control design for spacecraft under control input saturation," Journal of Guidance, Control, and Dynamics, vol. 27, no. 4, pp. 627-633, 2004.

[13] A.-M. Zou, A. H. J. de Ruiter, and K. D. Kumar, "Finite-time attitude tracking control for rigid spacecraft with control input constraints," IET Control Theory \& Applications, vol. 11, no. 7, pp. 931-940, 2017.

[14] H. Jinchang and Z. Honghua, "A simple saturated control framework for spacecraft with bounded disturbances," International Journal of Robust and Nonlinear Control, vol. 26, no. 3, pp. 367-384, 2015.

[15] H. Long, F. Liu, J. Zhao, X. Xia, and C. Zhu, "Anti-unwinding constrained attitude control for flexible spacecraft with actuator saturation," Proceedings of the Institution of Mechanical
Engineers, Part G: Journal of Aerospace Engineering, vol. 230, no. 1, pp. 90-104, 2015.

[16] Y. Jiang, Q. Hu, and G. Ma, "Adaptive backstepping faulttolerant control for flexible spacecraft with unknown bounded disturbances and actuator failures," ISA Transactions, vol. 49, no. 1, pp. 57-69, 2010.

[17] Q. Shen, B. Jiang, and V. Cocquempot, "Fuzzy logic systembased adaptive fault-tolerant control for near-space vehicle attitude dynamics with actuator faults," IEEE Transactions on Fuzzy Systems, vol. 21, no. 2, pp. 289-300, 2013.

[18] Q. Shen, D. Wang, S. Zhu, and E. K. Poh, "Integral-type sliding mode fault-tolerant control for attitude stabilization of spacecraft," IEEE Transactions on Control Systems Technology, vol. 23, no. 3, pp. 1131-1138, 2015.

[19] Q. Hu, B. Xiao, and M. I. Friswell, "Robust fault-tolerant control for spacecraft attitude stabilisation subject to input saturation," IET Control Theory \& Applications, vol. 5, no. 2, pp. 271-282, 2011.

[20] Y. Han, J. D. Biggs, and N. Cui, "Adaptive fault-tolerant control of spacecraft attitude dynamics with actuator failures," Journal of Guidance, Control, and Dynamics, vol. 38, no. 10, pp. 2033-2042, 2015.

[21] B. Xiao, Q. Hu, and Y. Zhang, "Adaptive sliding mode fault tolerant attitude tracking control for flexible spacecraft under actuator saturation," IEEE Transactions on Control Systems Technology, vol. 20, no. 6, pp. 1605-1612, 2012.

[22] A.-M. Zou and K. D. Kumar, "Adaptive fuzzy fault-tolerant attitude control of spacecraft," Control Engineering Practice, vol. 19, no. 1, pp. 10-21, 2011.

[23] C. Cheng, Y. Di, J. Xu, and T. Yuan, "Advanced backstepping control based on ADR for non-affine non-strict feedback nonlinear systems," Automatika, vol. 59, no. 2, pp. 220-230, 2018.

[24] C. H. Cheng, Y. HU, and J. WU, "Auto disturbance controller of non-affine nonlinear pure feedback systems," Acta Automatica Sinica, vol. 40, no. 7, pp. 1528-1536, 2014.

[25] C. Chunhua, H. Yunan, and W. Jinhua, "Auto disturbance rejection controller for non-affine nonlinear systems with adaptive observers," Control Theory \& Applications, vol. 31, no. 2, pp. 148-158, 2014.

[26] C. Chun-hua, W. Jin-hua, Y. Hu, and L. Jing, "Adaptive control of non-affine nonlinear 320 system with saturation constraint," Control Theory \& Applications, vol. 31, no. 8, pp. 1000-1008, 2014.

[27] K. Wang, F. Xiao, J. Pang, J. Ren, C. Duan, and L. Li, "State of charge (SOC) estimation of lithium-ion battery based on adaptive square root unscented kalman filter," International Journal of Electrochemical Science, vol. 15, no. 9, pp. 94999516, 2020.

[28] J. Han, Active Disturbance Rejection Control Technique-The Technique for Estimating and Compensating the Uncertainties, National Defense Industry Press, Beijing, China, 2008.

[29] M. Krstic, I. Kanellakopoulos, and V. Petar, Nonlinear and Adaptive Control Design, Wiley, New York, NY, USA, 1995.

[30] Q. Hu, "Robust adaptive sliding mode attitude maneuvering and vibration damping of three-axis-stabilized flexible spacecraft with actuator saturation limits," Nonlinear Dynamics, vol. 55, no. 4, pp. 301-321, 2008. 